\title{
Null Geodesics and Strong Field Gravitational Lensing in a String Cloud Background
}

\author{
M. Sharif ${ }^{1}$ and Sehrish Iftikhar ${ }^{1,2}$ \\ ${ }^{1}$ Department of Mathematics, University of the Punjab, Quaid-e-Azam Campus, Lahore 54590, Pakistan \\ ${ }^{2}$ Department of Mathematics, Lahore College for Women University, Lahore 54000, Pakistan \\ Correspondence should be addressed to M. Sharif; msharif.math@pu.edu.pk
}

Received 2 March 2015; Accepted 1 May 2015

Academic Editor: Juan José Sanz-Cillero

Copyright (C) 2015 M. Sharif and S. Iftikhar. This is an open access article distributed under the Creative Commons Attribution License, which permits unrestricted use, distribution, and reproduction in any medium, provided the original work is properly cited. The publication of this article was funded by SCOAP ${ }^{3}$.

This paper is devoted to studying two interesting issues of a black hole with string cloud background. Firstly, we investigate null geodesics and find unstable orbital motion of particles. Secondly, we calculate deflection angle in strong field limit. We then find positions, magnifications, and observables of relativistic images for supermassive black hole at the galactic center. We conclude that string parameter highly affects the lensing process and results turn out to be quite different from the Schwarzschild black hole.

\section{Introduction}

The study of null geodesics is interesting from both astrophysical and theoretical points of view. This phenomenon helps to understand geometrical structure of spacetime as well as explaining high energy phenomenon occurring near black hole $(\mathrm{BH})$ such as accretion disks where particles move in circular orbits and formation of jets in which particles escape. The dynamics of test particle is useful to understand the observational effects such as deflection of light, time delays, and perihelion shift. Chandrasekhar [1] did the pioneer work in the geodesic study of Schwarzschild, Reissner-Nordström, and Kerr BHs. There has been growing interest to explore the behavior of geodesics around $\mathrm{BHs}$ [2-5] in the last few years.

Deflection of light ray by massive objects near a gravitational field is one of the important results in general relativity (GR) known as gravitational lensing. The deflection angle of light depends on the nature as well as distance of lens to an observer. Gravitational lensing is an effective tool to test predictions of GR as well as to study nonluminous objects such as extra solar planets, distant stars, detection of dark matter, estimation of cosmological parameter, detection of gravitational waves, and cosmic censorship conjuncture [69].

There are two types of gravitational lensing: weak and strong field limit. Initially, it was believed that gravitational lensing is based only on weak field and small deflection angles. Weak field approximation is very useful for investigating the properties of stars and galaxies $[10,11]$. However, in the last decade, the gravitational lensing in strong field regime (testing the lensing properties near photon sphere) has gained much attention. The deflection of light in strong field provides a platform to test a theory of gravitation in its general form. Since, in weak field approximation, alternative gravitational theories must agree with GR, so it would be interesting to study strong field approximation to show deviations from GR [12]. The gravitational field around the collapsed objects (BHs and neutron stars) is very strong. An astrophysical system involving such objects provides a way to investigate its properties in the context of strong field limit. The accretion matter of BHs and neutron stars emits radiations which originate from deep gravitational fields (at a distance of gravitational radius $r_{g}=G M / c^{2}$ ). Thus the motion of accretion matter also motivates to study strong field gravity [13].

Virbhadra and Ellis [14] studied strong field gravitational lensing of Schwarzschild $\mathrm{BH}$. They found a sequence of relativistic images on both sides of optical axis due to large deflection of light near the photon sphere. Frittelli et al. [15] proposed an exact lens equation without background spacetime and showed that the thin lens equation in strong field is remarkably accurate; even light rays take several 
rounds around the lens before reaching the observer. Bozza [16] studied spherically symmetric BHs in strong field limit and expanded the deflection angle near photon sphere. $\mathrm{He}$ remarked that this method is valid for a generic spherically symmetric metric regardless of the field equations, assuming that the light follows geodesic equation.

There is no direct observational evidence of gravitational lensing by $\mathrm{BH}$ or other compact objects. The detection of images for small $\mathrm{BH}$ is difficult whereas supermassive BHs such as $\operatorname{Sgr} A^{*}$ provide a good example to test the bending of light in strong gravity regimes [17]. Many people [18-20] studied various aspects of gravitational lensing for rotating BHs. Eiroa and Sendra [21] explored Bardeen regular $\mathrm{BH}$ as gravitational lens and compared the results with Schwarzschild BH. Many astrophysical spacetimes such as fermion stars [22], naked singularities [23], magnetized [24] as well as alternative gravity BHs [25], and wormholes [26] are analyzed as gravitational lenses. Recently, Wei et al. [27] investigated gravitational lensing of Hayward $\mathrm{BH}$ and found that nonsingularity parameter has negligible effect in the weak field while it has a significant influence in the strong field limit.

Gravitational lensing for BHs in string and other higher dimensional theories has recently attained much attention. Bhadra [28] studied gravitational lensing of charged $\mathrm{BH}$ of heterotic string theory and found no significant string in strong field regime. Eiroa and Sendra [29] studied massless braneworld $\mathrm{BH}$ in weak as well as strong field and compared the results with Schwarzschild and Reissner-Nordström BHs. Tsukamoto et al. [30] examined lensing properties of Tangherlini spacetime and concluded that images have little effect on the total light curve in strong field.

In this paper, we study null geodesics as well as gravitational lensing of a spherically symmetric $\mathrm{BH}$ with string cloud background in strong field. The paper is organized as follows. In Section 2, we introduce metric for the string cloud and study the behavior of null geodesics. Section 3 evaluates exact deflection angle using Bozza method. In Section 4, we explore positions, magnifications, and observables of the relativistic images for the supermassive galactic $\mathrm{BH}$. In the last section, we conclude our results.

\section{Basic Equations and Null Geodesics}

Cosmic strings are considered as a generic outcome of symmetry breaking phase transitions of the early universe and play a vital role in the formation of large scale structure of the universe $[31,32]$. It is believed that strings may exist in the early universe and are very important in creation of density inhomogeneities [33]. String theory can describe many features of BHs. The association of strings with $\mathrm{BHs}$ is suggested by the relationship between entropy of $\mathrm{BH}$ horizon and string states [34]. We consider static spherically symmetric spacetime with string cloud background [35] as

$$
\begin{aligned}
d s^{2}= & -f(r) d t^{2}+f^{-1}(r) d r^{2} \\
& +g(r)\left(d \theta^{2}+\sin ^{2} \theta d \phi^{2}\right),
\end{aligned}
$$

where

$$
\begin{aligned}
& f(r)=\left(1-\frac{2 M}{r}-\omega\right), \\
& g(r)=r^{2} .
\end{aligned}
$$

$\omega$ is the string cloud parameter and $M$ is the mass of $\mathrm{BH}$ (independent of $\omega$ ). This metric represents $\mathrm{BH}$ related to spherical mass $M$ surrounded by a cloud of strings which can also be considered as a metric associated to a global monopole. The corresponding event horizon is

$$
r_{h}=\frac{2 M}{1-\omega} .
$$

In the limit $\omega \rightarrow 0$, the Schwarzschild radius is recovered while it approaches infinity when $\omega \rightarrow 1$. Thus, for a realistic model, $0<\omega<1$. Also, for $M=0$, it does not have any horizon and generates a naked singularity at $r=0$.

The Lagrangian in the equatorial plane $(\theta=\pi / 2)$ [1] for a photon traveling in string cloud is

$$
2 \mathscr{L}=-\left(1-\frac{2 M}{r}-\omega\right) \dot{t}^{2}+\frac{\dot{r}^{2}}{(1-2 M / r-\omega)}+r^{2} \dot{\phi} .
$$

Using the Euler-Lagrange equations for null geodesics and an affine parameter $l$, we have

$$
\begin{aligned}
& \dot{t}=\frac{d t}{d l}=\frac{E}{(1-2 M / r-\omega)}, \\
& \dot{\phi}=\frac{d t}{d l}=\frac{L}{r^{2}},
\end{aligned}
$$

where $E$ and $L$ are the energy and angular momentum per unit mass. The Hamiltonian is given as

$$
2 \mathscr{H}=-E \dot{t}+L \dot{\phi}+\frac{\dot{r}^{2}}{(1-2 M / r-\omega)}+r^{2} \dot{\phi}^{2}=\delta,
$$

where $\delta$ is an integral of motion and $\delta=-1,0,1$ correspond to spacelike, null, and time like geodesics, respectively. For null geodesics, the radial equation of motion is

$$
\dot{r}^{2}+V_{\text {eff }}(r)=E,
$$

where $V_{\text {eff }}=\left(L / r^{2}\right)(1-2 M / r-\omega)$. Figure 1 shows the behavior of effective potential for different values of $\omega(0<\omega<1)$ and angular momentum $L$. The dashed lines represent event horizons $\left(r_{h}=2.2,2.5,3.33,5\right.$ for $\left.\omega=0.1,0.2,0.4,0.6\right)$. We see that maximum values exist outside the event horizons. Since there does not exist any minimum value of $V_{\text {eff }}$, hence only unstable circular orbits exist.

\section{Deflection Angle}

In this section, following [16], we calculate deflection angle for (1) in the strong field limit. We consider photon sphere as an initial point which is the strong gravitational region in spacetime such that photons are forced to travel in their 


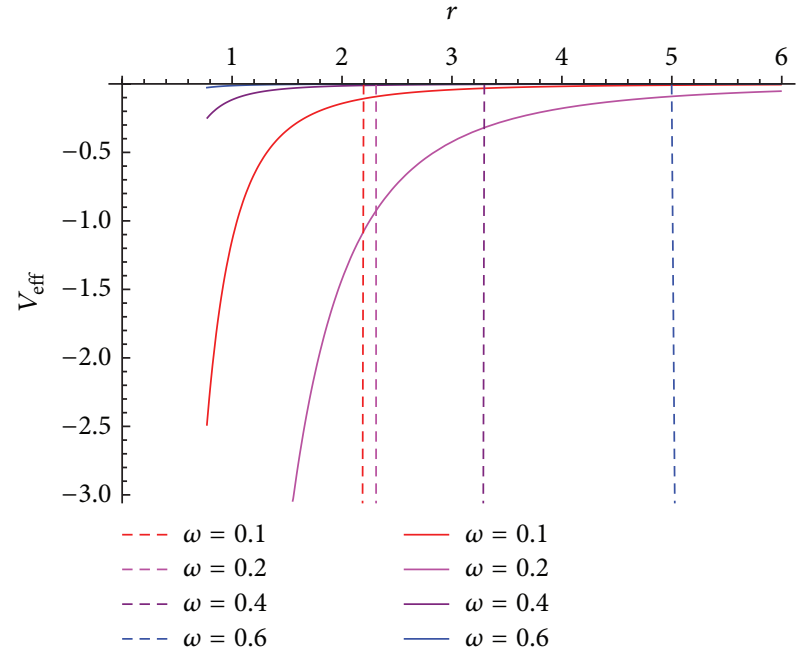

(a)

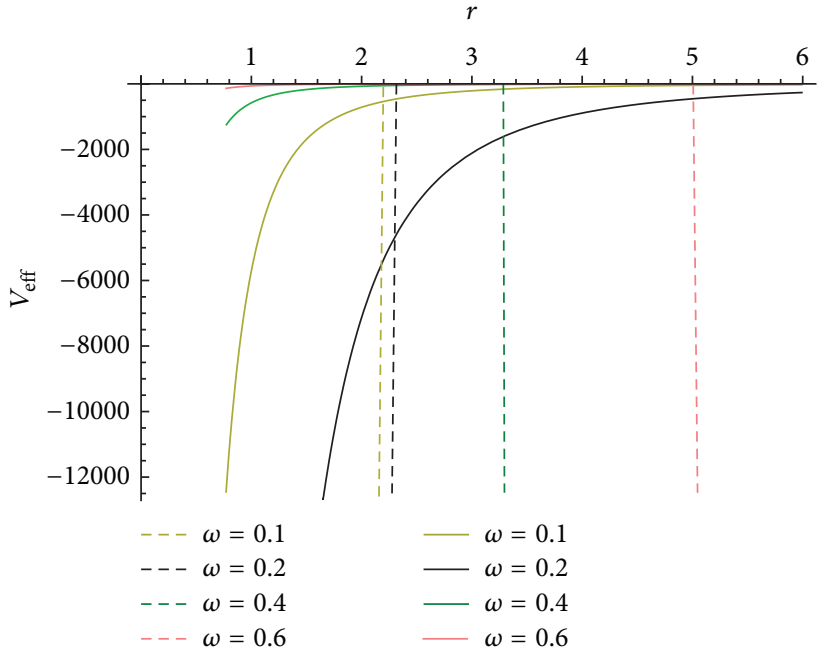

(b)

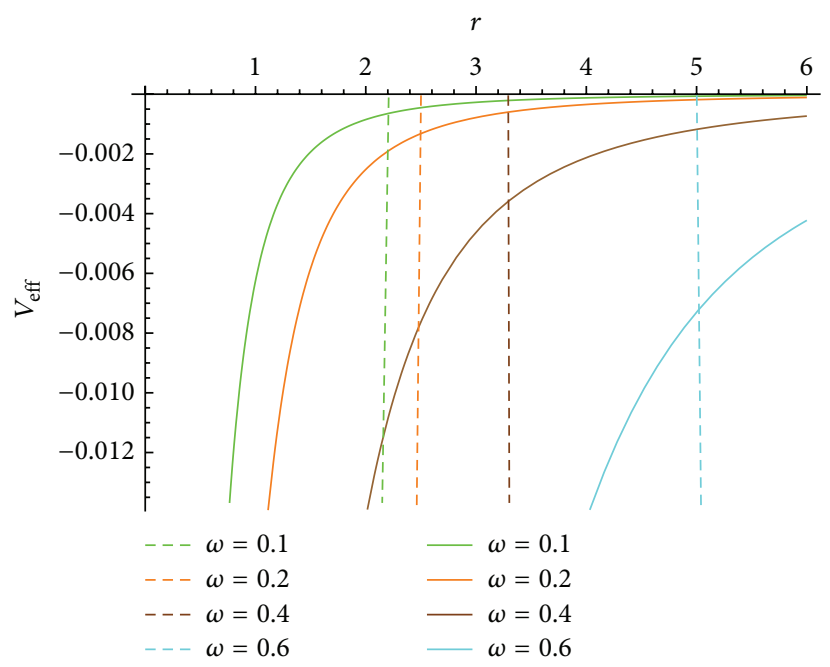

(c)

Figure 1: Behavior of the effective potential $\left(V_{\text {eff }}\right)$ versus $r$ corresponding to $L=3.22$ (a), $L=1$ (b), and $L=5$ (c) with $M=1$. Here, solid and dashed lines correspond to $V_{\text {eff }}$ and $r_{h}$.

orbits. It is useful to write (1) in terms of Schwarzschild radius by defining the transformations $x=r / 2 M, T=t / 2 M$ as

$$
\begin{aligned}
d s^{2}= & -f(x) d T^{2}+f^{-1}(x) d x^{2} \\
& +g(x)\left(d \theta^{2}+\sin ^{2} \theta d \phi^{2}\right),
\end{aligned}
$$

where

$$
\begin{aligned}
& f(x)=\left(1-\frac{1}{x}-\omega\right), \\
& g(x)=x^{2} .
\end{aligned}
$$

The radius of photon sphere $x_{\mathrm{ps}}$ is the largest positive root of the equation

$$
\frac{f^{\prime}(x)}{f(x)}=\frac{g^{\prime}(x)}{g(x)}
$$

and yields

$$
x_{\mathrm{ps}}=\frac{3}{2(1-\omega)} .
$$

The behavior of $x_{\mathrm{ps}}$ is shown in Figure 2 which increases with the increasing values of $\omega$ and is larger than that of the Schwarzschild radius. Also, for $\omega=0$, radius of photon sphere of string cloud coincides with that of Schwarzschild. The deflection angle for a photon (from infinity) is calculated as a function of distance of the closest approach (minimum distance) $x_{0}$ :

$$
\alpha\left(x_{0}\right)=I\left(x_{0}\right)-\pi,
$$

where

$$
I\left(x_{0}\right)=\int_{x_{0}}^{\infty} \frac{2 \sqrt{f^{-1}(x)} d x}{\sqrt{g(x)} \sqrt{f\left(x_{0}\right) g(x) / f(x) g\left(x_{0}\right)-1}} .
$$




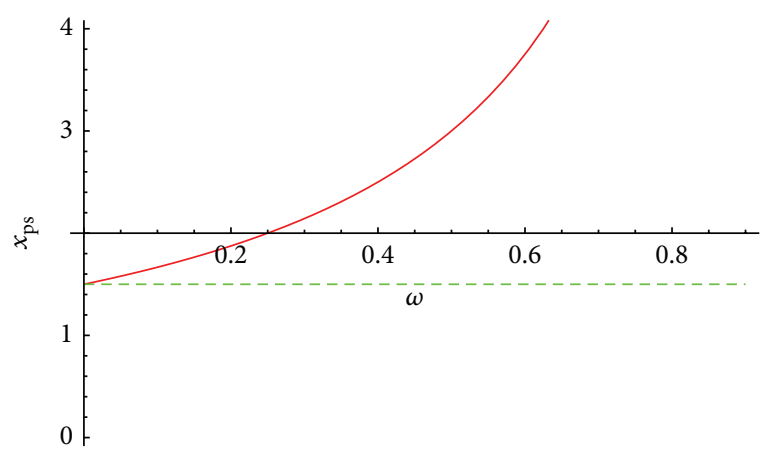

FIGURE 2: Radius of photon sphere $\left(x_{\mathrm{ps}}\right)$ for the string cloud versus string parameter $\omega$. Red curve corresponds to $x_{\mathrm{ps}}$ whereas the green line (dashed) indicates Schwarzschild limit.

The deflection angle depends upon the relation between $x_{0}$ and $x_{\mathrm{ps}}$ which grows if $x_{0}$ approaches $x_{\mathrm{ps}}$. Following [16], we define a new variable $z$ :

$$
z=\frac{f(x)-f\left(x_{0}\right)}{1-f\left(x_{0}\right)}=\frac{x-x_{0}}{x\left(1+\omega\left(x_{0}\right)\right)} .
$$

Replacing $z$ in (13), it follows that

$$
I\left(x_{0}\right)=\int_{0}^{1} R\left(z, x_{0}\right) h\left(z, x_{0}\right) d z,
$$

where

$$
\begin{aligned}
& R\left(z, x_{0}\right)=2 \frac{\sqrt{f(x) f^{-1}(x)}}{f^{\prime}\left(x_{0}\right) g(x)}\left(1-f\left(x_{0}\right)\right) \sqrt{g\left(x_{0}\right)}=2(1 \\
& \left.\quad+\omega x_{0}\right) \\
& h\left(z, x_{0}\right) \\
& \quad=\frac{1}{\sqrt{f\left(x_{0}\right)-\left[\left(1-f\left(x_{0}\right)\right) z+f\left(x_{0}\right)\right]\left(g\left(x_{0}\right) / g(x)\right)}} \\
& \quad=\left[z\left(2-\frac{3}{x_{0}}\right)+\omega\left(x_{0}(1-\omega)-5\right)\right. \\
& \quad+z^{2}\left(\frac{3}{x_{0}}-1+\omega\left(7-2 x_{0}\right)-\omega^{2} x_{0}\left(5-x_{0}\right)\right)+\omega^{3} x_{0}^{2} \\
& \left.\quad-z^{3}\left(\frac{1}{x_{0}}+3 \omega+3 \omega^{2} x_{0}+\omega^{3} x_{0}^{2}\right)\right]^{-1 / 2} .
\end{aligned}
$$

All functions other than the subscript 0 are evaluated at

$$
x=\frac{\left[1-f\left(x_{0}\right) z+f\left(x_{0}\right)\right]}{f(x)} .
$$

The function $R\left(z, x_{0}\right)$ is regular for all values of $z$ and $x_{0}$ while $h\left(z, x_{0}\right)$ diverges for $z \rightarrow 0$. Thus we expand this function inside the square root up to second order in $z$ using Taylor's series expansion as

$$
h_{0}\left(z, x_{0}\right)=\frac{1}{\sqrt{\zeta\left(x_{0}\right) z+\eta\left(x_{0}\right) z^{2}}}
$$

where

$$
\begin{aligned}
& \zeta\left(x_{0}\right)=\frac{1-f\left(x_{0}\right)}{f^{\prime}\left(x_{0}\right) g\left(x_{0}\right)}\left[f\left(x_{0}\right) g^{\prime}\left(x_{0}\right)-f^{\prime}\left(x_{0}\right)\right. \\
& \left.\cdot g\left(x_{0}\right)\right]=\frac{1+\omega x_{0}}{x_{0}}\left[2 x_{0}(1-\omega)-3\right] \\
& \eta\left(x_{0}\right)=\frac{\left[1-f\left(x_{0}\right)\right]^{2}}{2\left(f^{\prime}\left(x_{0}\right)\right)^{3} g^{2}\left(x_{0}\right)}\left[2 \left\{\left(f^{\prime}\left(x_{0}\right)\right)^{2} g^{\prime}\left(x_{0}\right)\right.\right. \\
& \cdot g\left(x_{0}\right)-f\left(x_{0}\right) f^{\prime \prime}\left(x_{0}\right) g\left(x_{0}\right) g^{\prime}\left(x_{0}\right)+g\left(x_{0}\right) \\
& \left.\left.\cdot f^{\prime}\left(x_{0}\right)\left(g\left(x_{0}\right) g^{\prime \prime}\left(x_{0}\right)-2\left(g^{\prime}\left(x_{0}\right)\right)^{2}\right)\right\}\right]=(1 \\
& \left.+\omega x_{0}\right)^{2}\left[\frac{3}{x_{0}}-1+\omega\right] .
\end{aligned}
$$

Using these values in (13), we have

$$
I\left(x_{0}\right)=I_{D}\left(x_{0}\right)+I_{R}\left(x_{0}\right),
$$

where

$$
\begin{aligned}
& I_{D}\left(x_{0}\right)=\int_{0}^{1} R\left(0, x_{\mathrm{ps}}\right) h_{0}\left(z, x_{0}\right) d z, \\
& I_{R}\left(x_{0}\right) \\
& \quad=\int_{0}^{1}\left\{R\left(z, x_{0}\right) h\left(z, x_{0}\right)-R\left(0, x_{\mathrm{ps}}\right) h_{0}\left(z, x_{0}\right)\right\} d z .
\end{aligned}
$$

When $\zeta$ is nonzero $\left(x_{0} \neq x_{\mathrm{ps}}\right)$, the order of divergence in $h_{0}$ is approximately $1 / \sqrt{z}$ while, for $\zeta$ to be zero $\left(x_{0}=\right.$ $\left.x_{\mathrm{ps}}\right)$, the divergence is $1 / z$. Thus $I_{D}$ is the term which contains divergence whereas $I_{R}$ is regular as the divergence is subtracted out [16]. The impact parameter $J$ is related to $x_{0}$ by the conservation of angular momentum:

$$
J=\sqrt{\frac{g\left(x_{0}\right)}{f\left(x_{0}\right)}} .
$$

Bozza [16] showed that the deflection angle has logarithmic divergence for the photons traveling near to photon sphere given as

$$
\alpha(u)=-a_{1} \ln \left(\frac{u}{u_{\mathrm{ps}}}-1\right)+a_{2}+O\left(u-u_{\mathrm{ps}}\right),
$$

where $J_{\mathrm{ps}}=\sqrt{x_{\mathrm{ps}}^{3} /\left(x_{\mathrm{ps}}(1-\omega)-1\right)}$ is the value of impact parameter at $x_{0}=x_{\mathrm{ps}}$ and

$$
\begin{aligned}
a_{1} & =\frac{R\left(0, x_{\mathrm{ps}}\right)}{2 \sqrt{\eta\left(x_{\mathrm{ps}}\right)}} \\
& =\frac{1+\omega x_{\mathrm{ps}}}{\sqrt{\left(1 / x_{\mathrm{ps}}\right)\left(1+\omega x_{\mathrm{ps}}\right)^{2}\left[3+x_{\mathrm{ps}}(1-\omega)\right]}}, \\
a_{2} & =-\pi+a_{R}+a_{1} \ln 2 \xi
\end{aligned}
$$




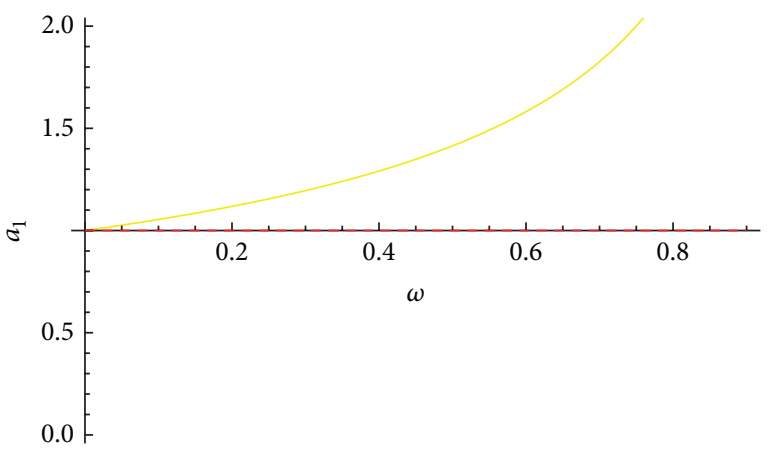

(a)

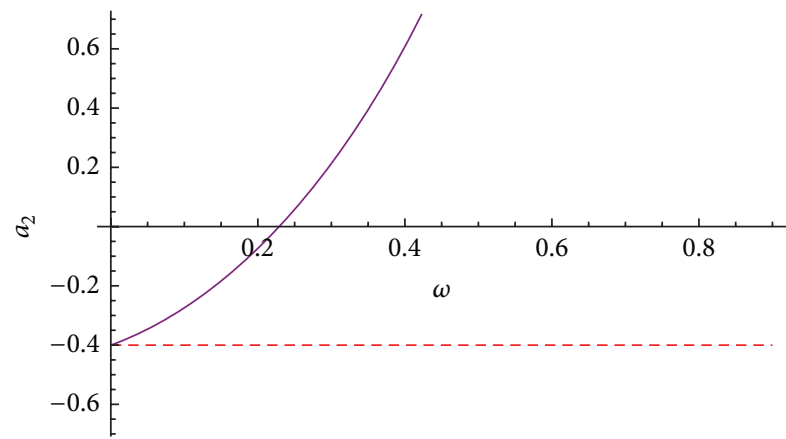

(b)

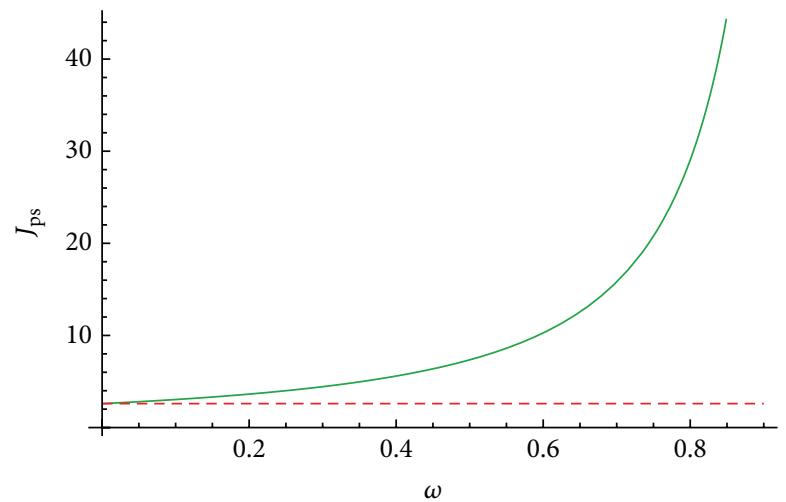

(c)

Figure 3: Behavior of strong field deflection coefficients $\left(a_{1}(\mathrm{a})\right.$ and $\left.a_{2}(\mathrm{~b})\right)$ and parameter $J_{\mathrm{ps}}(\mathrm{c})$ as functions of $\omega$ are represented by yellow, purple, and green while red (dashed) line shows Schwarzschild limit.

where

$$
\xi=\frac{2 \eta\left(x_{\mathrm{ps}}\right)}{A\left(x_{\mathrm{ps}}\right)}=\frac{2\left(1+\omega x_{\mathrm{ps}}\right)^{2}\left[3+x_{\mathrm{ps}}(\omega-1)\right]}{\left(x_{\mathrm{ps}}(1-\omega)-1\right)} .
$$

Since $a_{R}$ cannot be calculated directly, so we expand it using Taylor's series expansion up to second order in terms of $\omega$ as

$$
a_{R}=a_{R, 0}+a_{R, 2} \omega^{2}+O\left(\omega^{4}\right)
$$

where $a_{R, 0}$ corresponds to the uncharged (Schwarzschild) $\mathrm{BH}$ [16] and $a_{R, 2}=3 \ln (6(2-\sqrt{3})) \approx-0.708333$. The plots of $a_{1}, a_{2}$, and $J_{\mathrm{ps}}$ along with comparison of Schwarzschild BH are shown in Figure 3 . We see that $a_{1}$ increases positively with the increasing values of $\omega$ while $a_{2}$ is negative for small values of $\omega$ but it grows positively with large values of $\omega$. Both coefficients are greater than the Schwarzschild limit. The impact parameter $J_{\mathrm{ps}}$ is monotonically increasing function of $\omega$ and also greater than the Schwarzschild limit.

\section{Positions, Magnifications, and Observables}

In this section, we study positions, magnifications, and observables of the relativistic images in the strong field limit, which are directly related by the deflection angle using the lens equation. The detailed discussion is available in $[21,29]$; here we give a review of the basic equations and derive our results numerically. The lens equation helps to explore the bending of light by relating the image to source position and has the following form [14]:

$$
\tan \beta=\tan \theta-\frac{D_{d s}}{D_{s}}\{\tan (\alpha-\theta)+\tan \theta\} .
$$

The basic lens formalism is shown in Figure 4 which is helpful in understanding the lens equation. When objects are aligned, the lensing effects become more important; hence we consider the case in which $\beta$ and $\theta$ are small. The nonzero values of $\beta$ give two weak deflection images (primary and secondary) and two infinite sets of point relativistic images. The deflection angle from the first set (relativistic images) is $\alpha=\Delta \alpha_{n}+2 n \pi, n \in N$ and $0<\Delta \alpha_{n} \ll 1$. In this approximation, the lens equation can be written as [12]

$$
\beta=\theta-\frac{D_{d s}}{D_{s}} \Delta \alpha_{n}
$$

For the other set of images $\left(\alpha=-\Delta \alpha_{n}-2 n \pi\right)$, the quantity $\Delta \alpha_{n}$ is replaced by $-\Delta \alpha_{n}$ in the above equation. The deflection angle can be expressed in terms of angular position of the image $\theta$ and the lens-observer distance $D_{d}$. According to lens geometry $\left(J=D_{d} \sin \theta \approx D_{d} \theta\right)$, (23) can be written as

$$
\alpha(\theta)=-a_{1} \ln \left(\frac{D_{d} \theta}{J_{\mathrm{ps}}}-1\right)+a_{2} .
$$




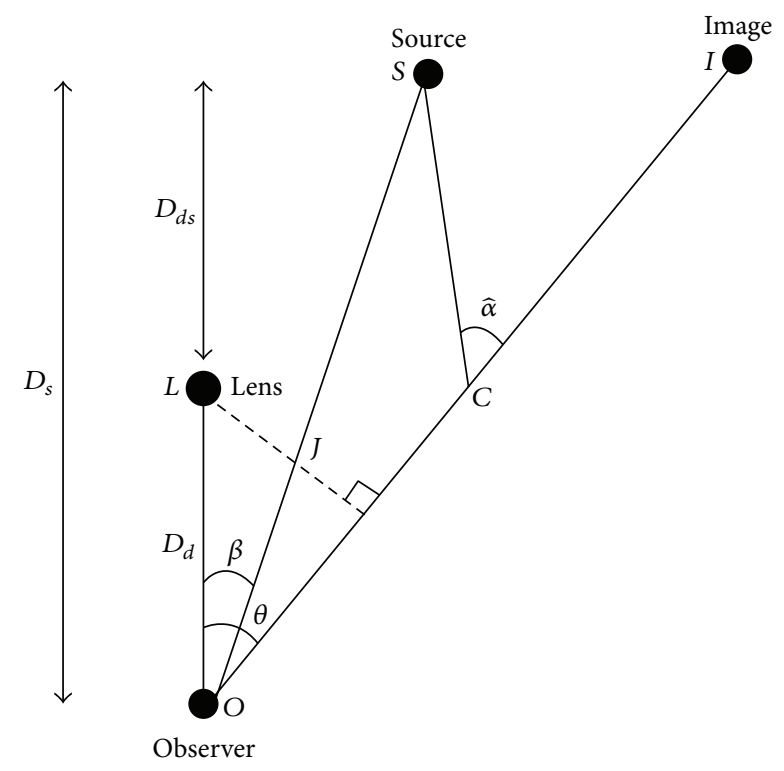

FIGURE 4: Basic lens formalism: position of source, image, lens, and observer are represented by $S, I, L$, and $O$. The distance between lens-source, lens-observer, and source-observer are denoted by $D_{d s}$, $D_{d}$, and $D_{s}$, respectively. The line connecting $O$ and $L$ is called optical axis of the lens geometry. The angle $\beta$ is the location of the source $S$, while the position of image is denoted by angle $\theta$ with respect to optical axis. The impact parameter is given by $J$ [36].

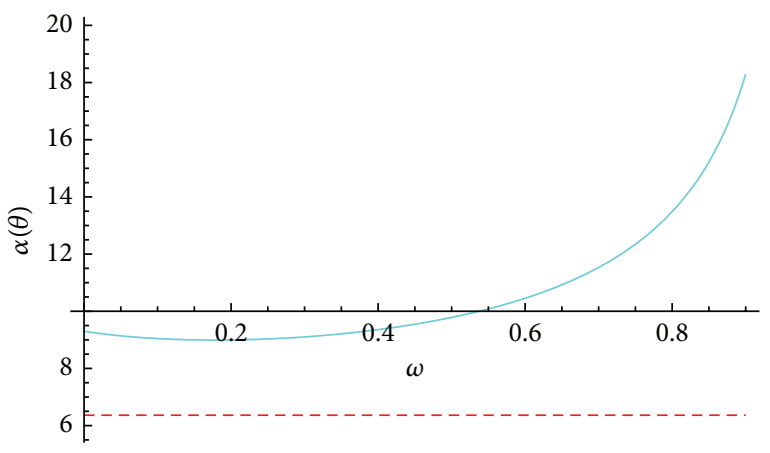

FIGURE 5: Deflection angle $\alpha$ for the string cloud is evaluated at $u=$ $u+0.03$ as a function of $\omega$. The Schwarzschild limit is shown by a horizontal dashed line.

Figure 5 indicates that deflection angle increases for light propagating in the strong field limit near a $\mathrm{BH}$ with string cloud background. We see that deflection angle is much larger than the Schwarzschild BH.

Inverting the above equation and using a first order Taylor's series expansion around $\alpha=2 n \pi$, one can obtain $n$th image position as

$$
\theta_{n}=\theta_{n}^{0}-\varsigma_{n} \Delta \alpha_{n}
$$

where

$$
\begin{aligned}
& \theta_{n}^{0}=\frac{J_{\mathrm{ps}}}{D_{d}}\left[1+e^{\left(a_{2}-2 n \pi\right) / a_{1}}\right], \\
& \varsigma_{n}=\frac{J_{\mathrm{ps}}}{a_{1} D_{d}} e^{\left(a_{2}-2 n \pi\right) / a_{1}} .
\end{aligned}
$$

Using (28) and (30), $\theta_{n}$ can be written as

$$
\theta_{n}=\theta_{n}^{0}-\frac{\varsigma_{n} D_{s}}{D_{d s}}\left(\theta_{n}-\beta\right)
$$

Assuming $0<\varsigma D_{s} / D_{d s} \ll 1$, the angular position of two sets of relativistic images are [21, 29]

$$
\begin{aligned}
& \theta_{n}=\theta_{n}^{0}+\frac{\varsigma D_{d}}{D_{d s}}\left(\beta-\theta_{n}^{0}\right), \\
& \theta_{n}=-\theta_{n}^{0}+\frac{\varsigma D_{d}}{D_{d s}}\left(\beta+\theta_{n}^{0}\right) .
\end{aligned}
$$

For $\beta=0$ in (33), an infinite sequence of Einstein rings is obtained as

$$
\theta_{n}^{E}=\left(1-\frac{\varsigma D_{d}}{D_{d s}}\right) \theta_{n}^{0}
$$

The magnification of $n$th relativistic image is given by the quotient of solid angles subtended by the image and source:

$$
\mu_{n}=\left|\frac{\sin \beta}{\sin \theta_{n}} \frac{d \beta}{d \theta_{n}}\right| .
$$

Keeping the angles small and using (33) and (36), the $n$th relativistic image can be written as

$$
\mu_{n}=\frac{1}{\beta}\left[\theta_{n}^{0}+\frac{\varsigma_{n} D_{s}}{D_{d s}}\left(\beta-\theta_{n}^{0}\right)\right] \frac{\varsigma_{n} D_{s}}{D_{d s}} .
$$

A first order approximation in $\varsigma_{n} D_{s} / D_{d s}$ gives

$$
\mu_{n}=\frac{1}{\beta} \frac{\theta_{n}^{0} \varsigma_{n} D_{s}}{D_{d s}} .
$$

When the first image is the outer most image with angular position $\theta_{1}$, then the angular separation of other relativistic images is

$$
\theta_{\infty}=\frac{J_{\mathrm{ps}}}{D_{d}},
$$

where $\theta_{\infty}$ is the asymptotic position of the set of relativistic images obtained in the limit $n \rightarrow \infty$. In the simple case, when only $\theta_{1}$ is resolved as a single image, the angular separation between first and limiting value of the set of images is

$$
s=\theta_{1}-\theta_{\infty},
$$

and ratio between the flux of first and sum of other images is

$$
r=\frac{\mu_{1}}{\sum_{n=2}^{\infty} \mu_{n}} .
$$



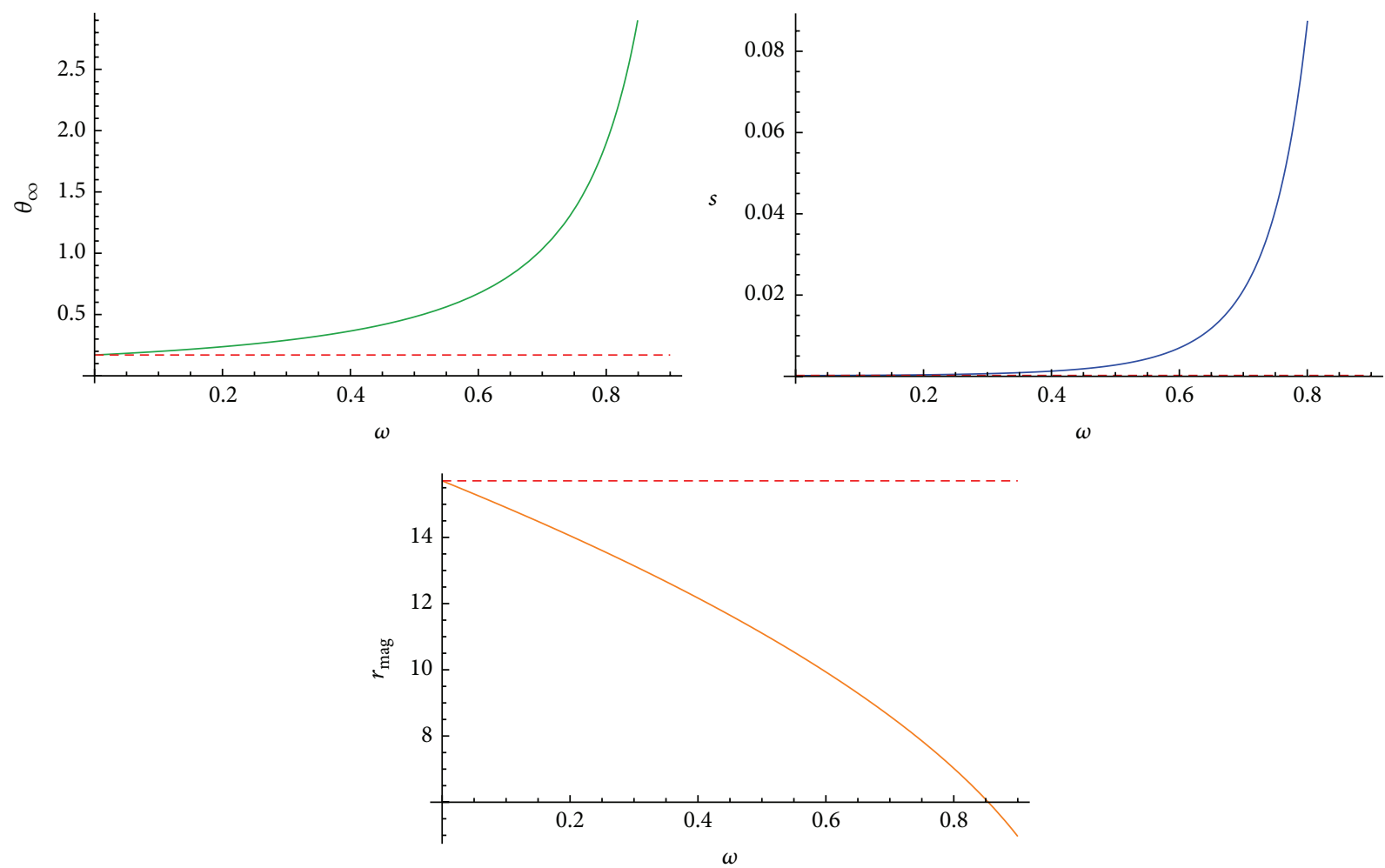

FIgURE 6: Plots of the observables corresponding to $\omega$. Dashed lines show the value for the Schwarzschild BH.

TABLE 1: Einstein rings and magnifications for $n=1$.

\begin{tabular}{cccccc}
\hline$\omega$ & 0.2 & 0.4 & 0.5 & 0.6 & 0.8 \\
\hline$\mu_{n}$ & 2.3879 & 2.7296 & 3.5033 & 5.53814 & 15.9764 \\
\hline$\theta_{n}^{E}$ & 0.2198 & 0.3654 & 0.4803 & 0.6713 & 1.4924 \\
\hline
\end{tabular}

TABLE 2: Einstein rings and magnifications for $n=2$.

\begin{tabular}{cccccc}
\hline$\omega$ & 0.2 & 0.4 & 0.5 & 0.6 & 0.8 \\
\hline$\mu_{n}$ & 2.1871 & 2.6006 & 3.1021 & 5.1683 & 12.4245 \\
\hline$\theta_{n}^{E}$ & 0.1961 & 0.3208 & 0.4575 & 0.5882 & 1.3437 \\
\hline
\end{tabular}

Using the concept of perfect alignment and the strong field limit, the observables can be written as

$$
\begin{aligned}
& s=\theta_{\infty} e^{\left(a_{2}-2 \pi\right) / a_{1}}, \\
& r=e^{2 \pi / a_{1}} .
\end{aligned}
$$

The existence of galactic supermassive BHs in our galaxy as well as in other galaxies is indicated by nuclear stellar dynamics $[37,38]$. We study the Einstein rings, magnifications, and observables in strong field limit for the supermassive BH. For the numerical example, we consider the supermassive $\mathrm{BH}$ in the center of galaxy NGC4486B, which has mass $5.7 \times 10^{8} \mathrm{M}_{\odot}$ and distance from the Earth $D_{d}=15.3 \mathrm{Mpc}$ [39]. For the sake of simplicity, we consider that the lens is placed midway between the source and observer; that is, $D_{d s} / D_{s}=1 / 2$ [14].

Tables 1 and 2 indicate results for the first $(n=1)$ and second image $(n=2)$. All the quantities have been found in
TABLE 3: Observables for the supermassive $\mathrm{BH}$ at the galactic center.

\begin{tabular}{lccccc}
\hline$\omega$ & 0.2 & 0.4 & 0.5 & 0.6 & 0.8 \\
\hline$\theta_{\infty}$ & 0.2373 & 0.3653 & 0.4802 & 0.6712 & 1.8985 \\
\hline$s$ & 0.0030 & 0.0061 & 0.0098 & 0.0170 & 0.0674 \\
\hline$r_{\text {mag }}$ & 16.9129 & 12.5487 & 10.7002 & 8.9718 & 5.5946 \\
\hline
\end{tabular}

$\mu a s$ and $r$ is converted into magnitudes $\left(r_{\operatorname{mag}}=2.5 \log r\right)$ [16]. We see that not only the number of Einstein rings increases with $\omega$ but also more Einstein rings $\left(\theta_{n}^{E}\right)$ are formed for the first image than the second one. The image magnification $\left(\mu_{n}\right)$ also shows increasing behavior for larger values of $\omega$ and images for $n=1$ are brighter than that of $n=2$. The graphical behavior of $\theta_{\infty}, s$, and $r_{\text {mag }}$ is shown in Figure 6 which indicates that, for the string cloud, magnification of the outer most image $r_{\text {mag }}$ is decreasing function of $\omega$ while the angular position $\theta_{\infty}$ and angular separation $s$ grow with the increase of $\omega$. It is also seen that $r_{\text {mag }}$ is smaller while $\theta_{\infty}$ and $s$ are larger than the Schwarzschild limit. Some numerical values of observables are presented in Table 3.

\section{Final Remarks}

In this paper, we have investigated null geodesics and gravitational lensing in the strong field limit for spherically symmetric $\mathrm{BH}$ with string cloud background. We have taken the example of supermassive $\mathrm{BH}$ in the center of galaxy NGC4486B for the numerical study and the results are compared with the Schwarzschild limit. We know that minimum 
value of $V_{\text {eff }}$ corresponds to stable while the maximum value represents that orbits are unstable. It is found that $V_{\text {eff }}$ attains only maximum values for different values of string parameter $\omega$ which indicates that the string parameter leads to unstable particle orbits.

Next, we have examined the behavior of light ray in the strong field regime for a string cloud as gravitational lens. For this purpose, we have evaluated deflection angle by using second order Taylor series expansion. The graphical analysis shows that, in the presence of string parameter $\omega$, the deflection angle grows positively and much greater than the Schwarzschild limit. The strong field deflection coefficients indicate that their behavior is different from the Schwarzschild $\mathrm{BH}$. We have also found numerical results for Einstein rings and magnifications (for the case of first and second image) as well as for three observables $\theta_{\infty}, s$, and $r_{\text {mag }}$ for supermassive $\mathrm{BH}$ in the center of galaxy. It is found that the number of Einstein rings as well as magnification of the relativistic images increases with the increase of $\omega$.

Finally, we have analysed numerically the inner most angular position of the relativistic images $\theta_{\infty}$, the angular separation $s$, and the relative magnitude $r_{\text {mag }}=2.5 \log r$. It is found that $r_{\text {mag }}$ diverge for large values of $\omega$ and smaller than the Schwarzschild limit while $\theta_{\infty}$ and $s$ increase rapidly and are large as compared to Schwarzschild $\mathrm{BH}$. The plots of $s$ show that the images are well separated for $\mathrm{BH}$ in a string cloud background than that of Schwarzschild BH. We conclude that the string parameter has a prominent effect on the motion of light in the strong field limit.

\section{Conflict of Interests}

The authors declare that they have no conflict of interests.

\section{References}

[1] S. Chandrasekhar, The Mathematical Theory of Black Holes, Oxford University Press, Oxford, UK, 1983.

[2] S. Fernando, D. Krug, and C. Curry, "Geodesic structure of static charged black hole solutions in $2+1$ dimensions," General Relativity and Gravitation, vol. 35, no. 7, pp. 1243-1261, 2003.

[3] C. Leiva, J. Saavedra, and J. Villanueva, "Geodesic structure of the schwarzschild black hole in rainbow gravity," Modern Physics Letters A, vol. 24, no. 18, pp. 1443-1451, 2009.

[4] S. Guha and P. Bhattacharya, "Geodesic motions near a fivedimensional Reissner-Nordström anti-de Sitter black hole," Journal of Physics: Conference Series, vol. 405, Article ID 012017, 2012.

[5] N. Cruz, M. Olivares, and J. R. Villaueva, "Geodesic structure of Lifshitz black holes in $2+1$ dimensions," The European Physical Journal C, vol. 73, article 2485, 2013.

[6] W. Sutherland, "Gravitational microlensing - a report on the MACHO project," Reviews of Modern Physics, vol. 71, no. 1, pp. 421-434, 1999.

[7] V. Perlick, "Gravitational lensing from a spacetime perspective," Living Reviews in Relativity, vol. 7, p. 9, 2004.

[8] I. Z. Stefanov, S. S. Yazadjiev, and G. G. Gyulchev, "Connection between black-hole quasinormal modes and lensing in the strong deflection limit," Physical Review Letters, vol. 104, Article ID 251103, 2010.
[9] S. W. Wei, Y. X. Liu, and H. Guo, "Relationship between highenergy absorption cross section and strong gravitational lensing for black hole," Physical Review D, vol. 84, no. 4, Article ID 041501R, 6 pages, 2011.

[10] P. Schneider, J. Ehlers, and E. E. Falco, Gravitational Lenses, Springer, 1992.

[11] R. D. Blandford and R. Narayan, "Cosmological applications of gravitational lensing," Annual Review of Astronomy and Astrophysics, vol. 30, pp. 311-358, 1992.

[12] V. Bozza, S. Capozziello, G. Iovane, and G. Scarpetta, "Strong field limit of black hole gravitational lensing," General Relativity and Gravitation, vol. 33, no. 9, pp. 1535-1548, 2001.

[13] A. Maselli, L. Gualtieri, P. Pani, L. Stella, and V. Ferrari, "Testing gravity with Quasi periodic oscillations from accreting black holes: the case of Einstein-Dilaton-Gauss-Bonnet theory," http://arxiv.org/abs/1412.3473.

[14] K. S. Virbhadra and G. F. R. Ellis, "Schwarzschild black hole lensing," Physical Review D, vol. 62, Article ID 084003, 2000.

[15] S. Frittelli, T. P. Kiling, and E. T. Newman, "Spacetime perspective of Schwarzschild lensing," Physical Review D, vol. 61, Article ID 064021, 2000.

[16] V. Bozza, "Gravitational lensing in the strong field limit," Physical Review D, vol. 66, Article ID 103001, 2002.

[17] A. Y. Bin-Nun, "Strong gravitational lensing by Sgr A"," Classical and Quantum Gravity, vol. 28, no. 11, Article ID 114003, 12 pages, 2011.

[18] A. de Vries, "The apparent shape of a rotating charged black hole, closed photon orbits and the bifurcation set $\mathrm{A}_{4}$," Classical and Quantum Gravity, vol. 17, no. 1, pp. 123-144, 2000.

[19] G. V. Kraniotis, "Gravitational lensing and frame dragging of light in the Kerr-Newman and the Kerr-Newman (anti) de Sitter black hole spacetimes," General Relativity and Gravitation, vol. 46, no. 11, article 1818, 2014.

[20] S. Chakraborty and A. K. Sen, "Light defection due to a charged, rotating body," http://arxiv.org/abs/1406.1295.

[21] E. F. Eiroa and C. M. Sendra, "Gravitational lensing by a regular black hole," Classical and Quantum Gravity, vol. 28, no. 8, Article ID 085008, 2011.

[22] N. Bilić, H. Nikolić, and R. D. Viollier, "Fermion stars as gravitational lenses," The Astrophysical Journal, vol. 537, no. 2, p. 909, 2000.

[23] G. Gyulchev and S. Yazadjiev, "Gravitational lensing by rotating naked singularities in the equatorial plane," AIP Conference Proceedings, vol. 94, pp. 106-118, 2007.

[24] R. A. Konoplya, "Magnetised black hole as a gravitational lens," Physics Letters B, vol. 644, no. 4, pp. 219-223, 2007.

[25] T. Kitamura, K. Nakajima, and H. Asada, "Demagnifying gravitational lenses toward hunting a clue of exotic matter and energy," Physical Review D, vol. 87, Article ID 027501, 2013.

[26] P. K. F. Kuhfittig, "Gravitational lensing of wormholes in the galactic halo region," The European Physical Journal C, vol. 74, no. 3, article 2818, 2014.

[27] S.-W. Wei, Y.-X. Liu, and C.-E. Fu, "Null geodesics and gravitational lensing in a nonsingular spacetime," Advances in High Energy Physics, vol. 2015, Article ID 454217, 11 pages, 2015.

[28] A. Bhadra, "Gravitational lensing by a charged black hole of string theory," Physical Review D, vol. 67, no. 10, Article ID 103009, 2003.

[29] E. F. Eiroa and C. M. Sendra, "Gravitational lensing by massless braneworld black holes," Physical Review D, vol. 86, Article ID 083009, 2012. 
[30] N. Tsukamoto, T. Kitamura, K. Nakajima, and H. Asada, "Gravitational lensing in Tangherlini spacetime in the weak gravitational field and the strong gravitational field," Physical Review D, vol. 90, Article ID 064043, 2014.

[31] T. W. B. Kibble, "Topology of cosmic domains and strings," Journal of Physics A: General Physics, vol. 9, no. 8, pp. 1387-1398, 1976.

[32] A. Vilenkin, "Cosmological density fluctuations produced by vacuum strings," Physical Review Letters, vol. 46, no. 17, pp. 1169$1172,1981$.

[33] D. Mitchell and N. Turok, "Statistical properties of cosmic strings," Nuclear Physics B, vol. 294, no. 4, pp. 1138-1163, 1987.

[34] A. Strominger and C. Vafa, "Microscopic origin of the Bekenstein-Hawking entropy," Physics Letters B, vol. 379, no. 14, pp. 99-104, 1996.

[35] P. S. Letelier, "Clouds of strings in general relativity," Physical Review D, vol. 20, no. 6, pp. 1294-1302, 1979.

[36] S. Sahu, M. Patil, D. Narasimha, and P. S. Joshi, "Can strong gravitational lensing distinguish naked singularities from black holes?" Physical Review D, vol. 86, no. 6, Article ID 063010, 13 pages, 2012.

[37] D. Richstone, E. A. Ajhar, R. Bender et al., "Supermassive black holes and the evolution of galaxies," Nature, vol. 395, p. A14, 1998.

[38] J. Magorrian, S. Tremaine, D. Richstone et al., "The demography of massive dark objects in galaxy centers," Astronomical Journal, vol. 115, no. 6, pp. 2285-2305, 1998.

[39] E. F. Eiroa and G. E. Romero, "Gravitational lensing of transient neutrino sources by black holes," Physics Letters B, vol. 663, no. 5, pp. 377-381, 2008. 

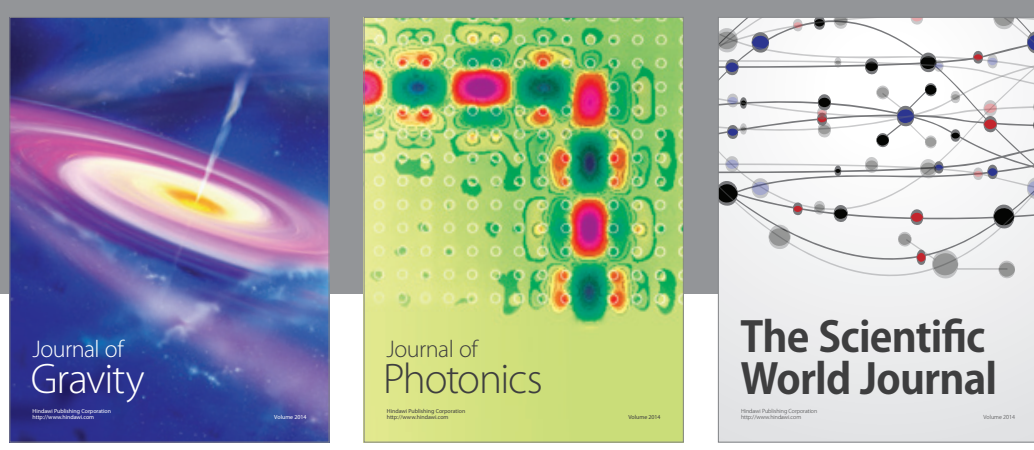

The Scientific World Journal
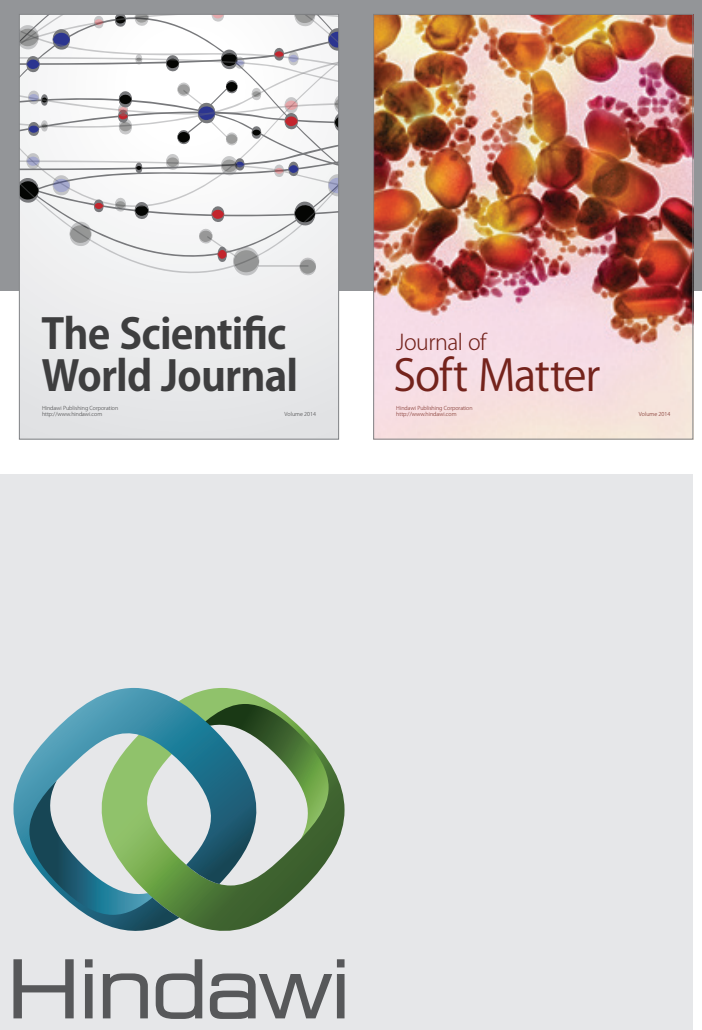

Submit your manuscripts at

http://www.hindawi.com

nternational Journal of

Statistical Mechanics
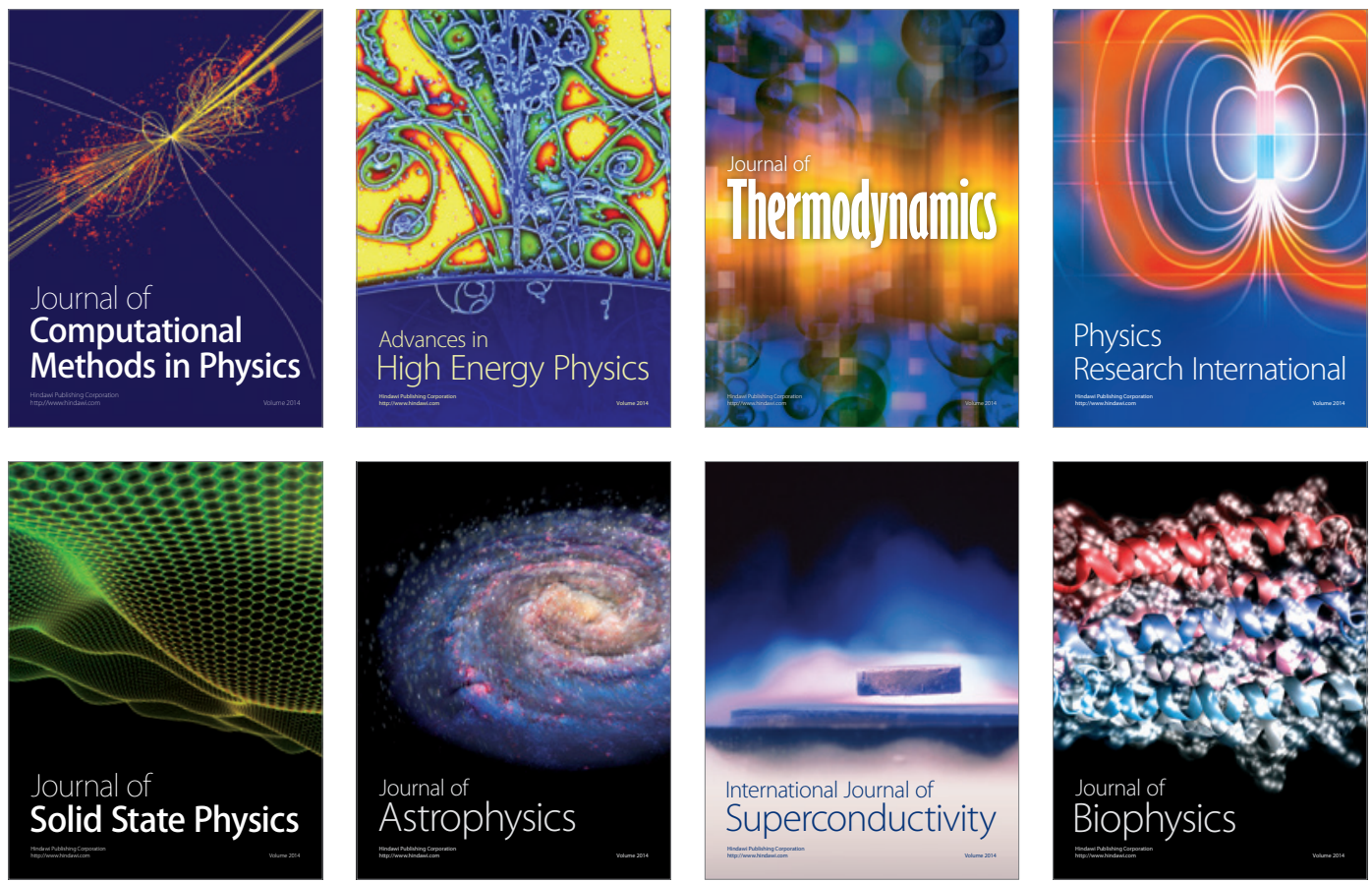
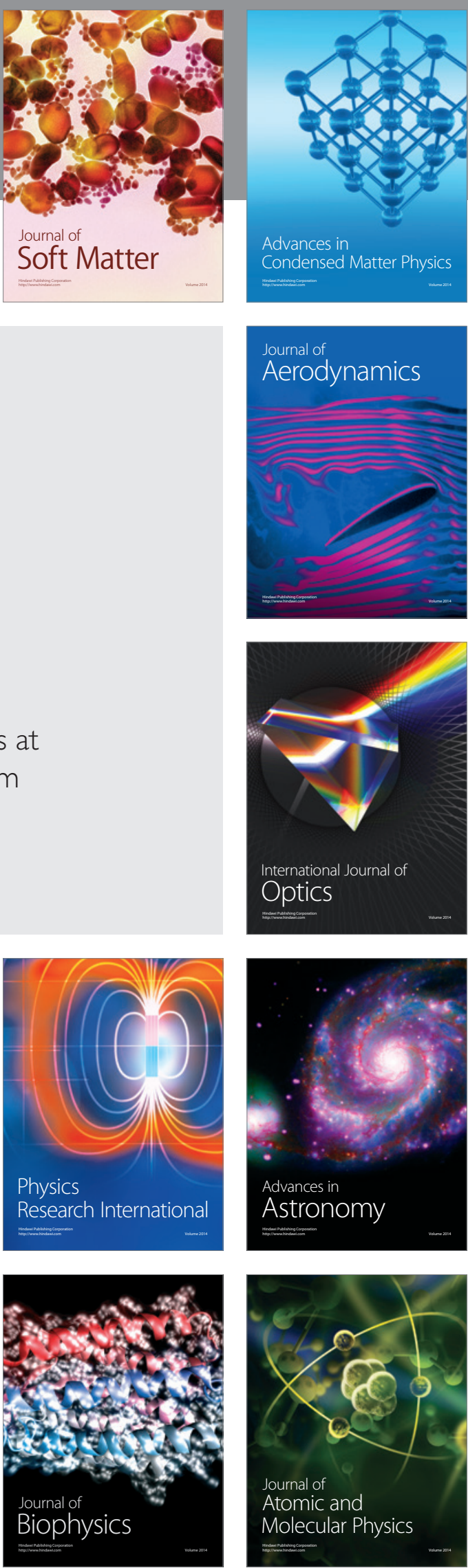